\title{
Proposta de sequenciamento das projeções mielográficas na identificação de compressão da medula espinhal em cães com doença do disco intervertebral cervical
}

\author{
[Sequential proposal in the myelographic views for detection of spinal cord compression \\ in dogs with cervical intervertebral disc disease] \\ M.L. Schwab ${ }^{1}$, A. Ripplinger ${ }^{1}$, G. Aiello ${ }^{1}$, D.A. Ferrarin ${ }^{1}$, A.C. Colvero ${ }^{1}$, \\ J. Rauber ${ }^{2}$, S. Simon', M.R. Wrzesinski², A. Mazzanti ${ }^{3 *}$ \\ ${ }^{1}$ Aluno de pós-graduação - Universidade Federal de Santa Maria (UFSM) - Santa Maria, RS \\ ${ }^{2}$ Aluno de graduação - Universidade Federal de Santa Maria (UFSM) - Santa Maria, RS \\ ${ }^{3}$ Universidade Federal de Santa Maria (UFSM) - Santa Maria, RS
}

\begin{abstract}
RESUMO
O objetivo deste trabalho foi verificar em quais projeções foi possível identificar compressão da medula espinhal em cães com doença do disco intervertebral (DDIV) cervical e propor um sequenciamento das projeções a ser realizado no exame mielográfico dessa região. Foram avaliadas quatro projeções mielográficas (lateral, ventrodorsal e oblíquas esquerda e direita) de 41 pacientes diagnosticados com DDIV cervical. Em 40 pacientes $(97,5 \%)$, foi possível identificar compressão da medula espinhal na projeção lateral; em 22 (53,6\%), nas oblíquas; e em $11(26,8 \%)$, na ventrodorsal $(\mathrm{P}<0,05)$. Havia lateralização da compressão em $22(53,6 \%)$ pacientes; $100 \%$ delas $(\mathrm{n}=22)$ foram detectadas pelas projeções oblíquas e $50 \%$ $(n=11)$ pela ventrodorsal. Em $10(24,4 \%)$ cães, foi observado mais que um local de compressão, tendo as projeções ventrodorsal e oblíquas auxiliado na definição do local de compressão em $50 \%$ e $70 \%$, respectivamente. Pode-se concluir que todas as projeções mielográficas estudadas permitem identificar compressão na medula espinhal em cães com DDIV cervical, sendo a incidência lateral a que mais a revelou, seguida das oblíquas e da ventrodorsal, estabelecendo-se, assim, uma proposta de sequenciamento das projeções mielográficas a serem realizadas para essa região.
\end{abstract}

Palavras-chave: cão, compressão, doença do disco intervertebral, mielografia, cirurgia

\begin{abstract}
The aim of this study was to verify in which of the myelographic views it was possible to identify spinal cord compression in dogs with cervical intervertebral disc disease (IVDD), and to establish a sequence in which myelographic views should be obtained for this region. Four myelographic views (lateral, ventrodorsal, left oblique and right oblique) of 41 patients diagnosed with cervical IVDD were evaluated. In 40 patients (97.5\%) it was possible to identify spinal cord compression by lateral view, 22 (53.6\%) by the oblique view, and $11(26.8 \%)$ by the ventrodorsal view $(P<0.05)$. There were lateralized compressions in $22(53.6 \%)$ patients, detected by all oblique views (100\%) and by 11 (50\%) of the ventrodorsal views. In $10(24.4 \%)$ dogs, more than one compression site was observed, where the ventrodorsal view helped to decide the site in 50\% of the cases and oblique in 70\%. It can be concluded that all the tested myelographic views allow the identification of spinal cord compressions in dogs with cervical IVDD, the lateral view being the most relevant, followed by the oblique and ventrodorsal view, therefore establishing a sequence of myelographic views should be obtained for this region.
\end{abstract}

Keywords: dog, compression, intervertebral disk disease, myelography, surgery

Recebido em 24 de agosto de 2018

Aceito em 24 de julho de 2019

*Autor para correspondência (corresponding author)

E-mail: alexamazza@yahoo.com.br 


\section{INTRODUÇÃO}

A doença do disco intervertebral (DDIV) é a principal causa de mielopatia compressiva em cães e afeta principalmente raças condrodistróficas, como Dachshund, Poodle Toy, Pequinês, Beagle, Lhasa Apso, Shih-Tzu e Cocker Spaniel (Griffin et al., 2009; Brisson, 2010). Pode envolver qualquer segmento da medula espinhal, sendo as regiões cervical (C1-C5) e toracolombar (T3-L3) as mais representativas (Brisson, 2010). A DDIV pode ser classificada em tipo I ou extrusão e II ou protrusão (Hansen et al., 2017). Os sinais clínicos, quando a doença envolve a região cervical, variam desde hiperestesia cervical até tetraparesia não ambulatória (Brisson, 2010).

O diagnóstico presuntivo de DDIV cervical tem como base o histórico, os sinais clínicos e os exames neurológico e de auxílio ao diagnóstico. Entre os exames complementares de imagem para identificação de compressões na medula espinhal ocasionadas pela DDIV, pode-se citar a mielografia, a tomografia computadorizada (TC) e a ressonância magnética (RM) (Brisson, 2010; Bos et al., 2012). Mesmo sendo considerada menos sensível que a TC e a RM, ainda existem estudos que indicam a mielografia como técnica de imagem na rotina clínica (Prince e Ortiz, 2017).

As projeções mielográficas geralmente empregadas são a lateral e a ventrodorsal, o que pode dificultar a deteç̧ão de compressões lateralizadas na medula espinhal (Olby et al., 1994; McCartney, 1997). Contudo, recentes estudos demonstraram que as projeções oblíquas foram imprescindíveis para a localização de lesões compressivas lateralizadas em cães com DDIV toracolombar, auxiliando o cirurgião na definição do lado de acesso cirúrgico à medula espinhal (Tanaka et al., 2004; Gibbons et al., 2006; Zardo et al., 2010). Para compressões cervicais, no entanto, há escassez de dados, e apenas um estudo demonstrou a importância das projeções oblíquas (Felts e Prata, 1983).

Além disso, Gibbons et al. (2006) estabeleceram um sequenciamento das projeções mielográficas a serem realizadas para a região toracolombar. Entretanto, segundo o conhecimento dos autores, até o momento nenhum trabalho foi encontrado padronizando a sequência das projeções para a região cervical.
Portanto, pela falta de estudo sobre projeções mielográficas na detecção de compressões na medula espinhal cervical em cães com DDIV e pela não padronização no sequenciamento dessas para a região cervical, o objetivo deste trabalho foi verificar em quais projeções (lateral, ventral, oblíquas direita e esquerda) foi possível identificar compressão da medula espinhal em cães com doença do disco intervertebral (DDIV) cervical e propor um sequenciamento dessas projeções durante a mielografia.

\section{MATERIAL E MÉTODOS}

Foram incluídos cães com diagnóstico definitivo de DDIV cervical tipo I (extrusão) e tipo II (protrusão) atendidos pelo Setor de Neurologia e Neurocirurgia Veterinária (SNNV) de uma Instituição de Ensino Superior, no período de maio de 2008 a fevereiro de 2018. Como critério de inclusão, foram selecionados somente cães que apresentavam ficha clínica completa (histórico, exame físico e neurológico), mielografia com identificação da linha de contraste nas quatro projeções (lateral, ventrodorsal e oblíquas direita e esquerda) e que foram submetidos ao procedimento cirúrgico de descompressão da medula espinhal.

A mielografia foi realizada conforme técnica descrita por Sharp e Wheeler (2005), com o paciente em decúbito lateral por punção na cisterna magna, sob anestesia geral inalatória. $\mathrm{O}$ contraste utilizado foi o iohexol $(300 \mathrm{mg} / \mathrm{mL}$ Omnipaque $^{\circledR}$ ), na dose de $0,3 \mathrm{ml} / \mathrm{kg}$ (Gibbons et al., 2006).

As projeções lateral, ventrodorsal e oblíquas direita e esquerda foram realizadas de forma aleatória, logo após a injeção do contraste. Para a realização da projeção ventrodorsal, os pacientes foram extubados com o intuito de evitar a sobreposição do traqueotubo no canal vertebral (Aeschbacher, 2010). Já para as projeções oblíquas, os pacientes foram angulados 30 graus em relação à mesa (Gibbons et al., 2006). O foco dos raios $\mathrm{X}$ foi centrado na área de suspeita da lesão, com base no exame neurológico e no exame radiográfico simples (Sharp e Wheeler, 2005).

As imagens que estavam em sistema analógico foram digitalizadas por meio de scanner comercial Hewlett-Packard $^{\circledR}$ (HP), modelo M 1120, conforme metodologia descrita por 
Davidson et al. (2001). O adaptador de transparência foi substituído por negatoscópio posicionado sobre o scanner (Higgins, 1997). As avaliações foram realizadas em apenas um computador, a fim de não haver discrepância quanto à qualidade de imagem pela diferença entre as configurações de tela.

As imagens foram avaliadas, de forma cega e aleatória, por três médicos veterinários $(\mathrm{A} 1, \mathrm{~A} 2 \mathrm{e}$ A3) com pelo menos dois anos de experiência em observações de mielografias. As projeções mielográficas foram consideradas diagnóstico quando havia desvio da linha de contraste no espaço subaracnoideo (Brisson, 2010) em pelo menos uma das projeções. Nos casos em que havia mais de uma compressão, os avaliadores escolheram a mais evidente, com base na falta de preenchimento ou de desvio da linha de contraste e na persistência da compressão em mais de uma projeção.

A análise estatística foi executada pelo programa Statistical Analysis System ${ }^{\circledR}$ (SAS), versão 9.2, com nível de significância $\mathrm{P}<0,05$. O teste binominal foi utilizado para definir qual projeção mielográfica foi mais relevante para o diagnóstico de DDIV cervical para os avaliadores. Para determinar a concordância entre os avaliadores, foi utilizado o teste Kappa. Segundo Landis e Koch (1977), Kappa<0 indica sem concordância; entre 0 e 0,19, fraca concordância; 0,2 e 0,39, concordância razoável; 0,4 e 0,59, moderada concordância; 0,6 e 0,79, forte concordância; 0,8 e 1,0 , concordância quase perfeita.

\section{RESULTADOS}

Foram encontrados 62 registros de pacientes que realizaram exame de mielografia cervical e cirurgia descompressiva com diagnóstico definitivo de DDIV cervical (Hansen tipo I e II). Desses, somente 41 pacientes atenderam os critérios preestabelecidos de inclusão.

A população estudada foi representada por $51,2 \%$ $(n=21)$ de cães da raça Dachshund. Outras raças incluíram Beagle $(n=5)$, Pequinês $(n=3)$, Dálmata $(\mathrm{n}=2)$, Labrador, Maltês, Malamute, Pug, e Yorkshire Terrier $(n=1)$. Cinco cães não tinham raça definida. A idade variou entre três e 15 anos, sendo a média das idades de 8,3 anos ( $\pm 2,74)$. Quanto ao gênero, 63,4\% $(n=26)$ dos cães eram machos e $36,6 \%(n=17)$, fêmeas.

O local de compressão variou de C2-C3 a C6-C7, sendo $22 \%(\mathrm{n}=9)$ das compressões localizadas entre C2-C3; 24,4\% ( $\mathrm{n}=10)$ entre C3-C4; 24,4\% $(\mathrm{n}=10)$ entre $\mathrm{C} 4-\mathrm{C} 5 ; 24,4 \%(\mathrm{n}=10)$ entre C5-C6; e 4,8\% (n=2) entre C6-C7 (Fig. 1); 92,7\% (n=38) das compressões eram extrusões (Hansen tipo I), e 7,3\% (n=3) eram protrusões (Hansen tipo II).

Os avaliadores identificaram compressão na medula espinhal pelas projeções lateral, oblíquas e ventrodorsal em $97,5 \%(n=40), 53,6 \%(n=22)$ e $26,8 \%(\mathrm{n}=11)$, respectivamente (Fig. 2). A projeção lateral foi a que mais identificou compressão da medula espinhal quando comparada com a ventrodorsal e as oblíquas $(\mathrm{P}<0,05)$.

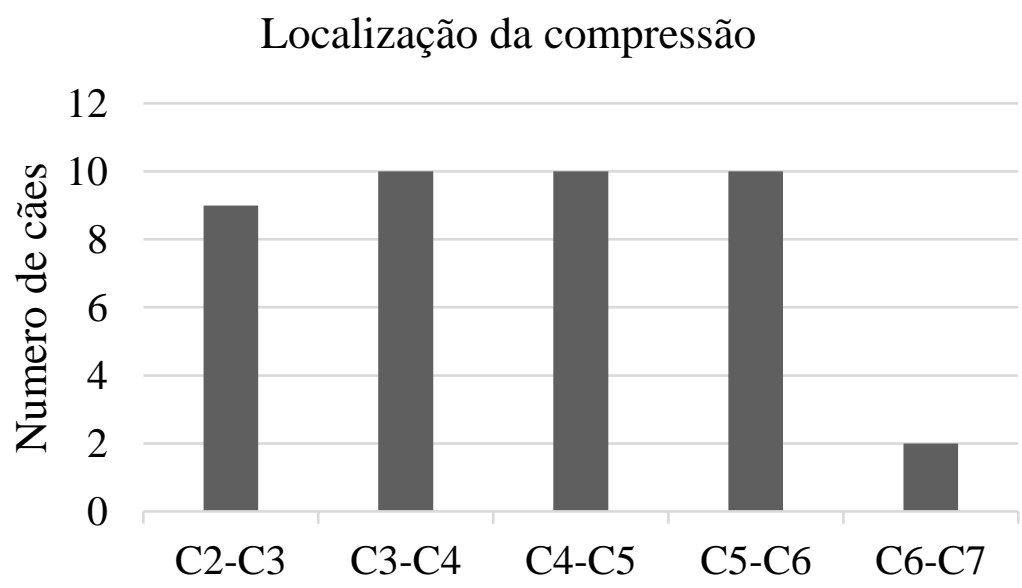

Figura 1. Distribuição quanto à localização da compressão identificada pela mielografia em cães com doença do disco intervertebral cervical. 

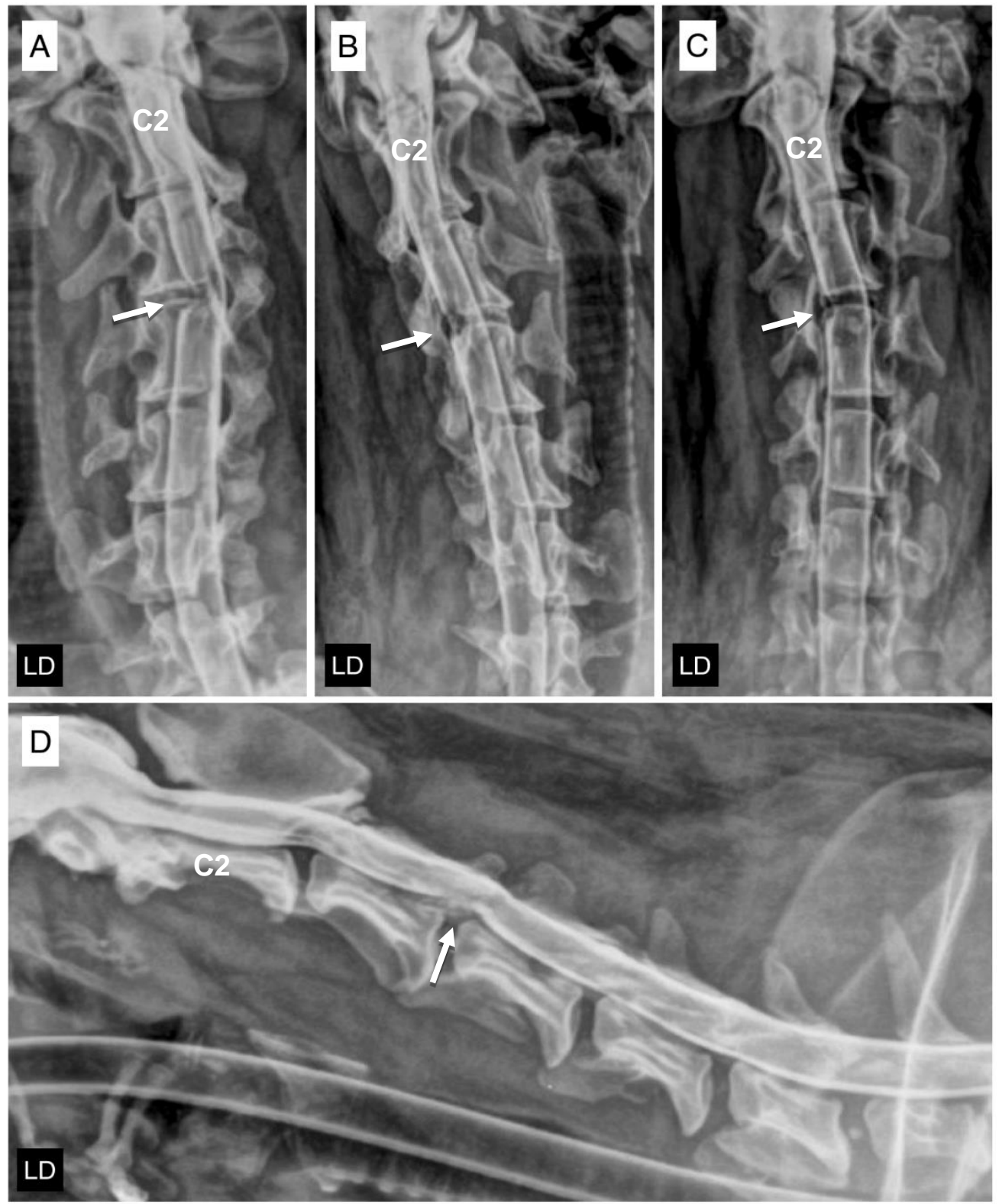

Figura 2. Mielografia em um cão com doença do disco intervertebral cervical (extrusão) nas projeções oblíqua direita (A), oblíqua esquerda (B), ventrodorsal (C) e lateral (D). Nota-se compressão entre C3-C4 identificada em todas as projeções mielográficas (setas). LD: lado direito.

Foi detectada lateralização da compressão em $53,6 \%(n=22)$ dos pacientes; $100 \%$ delas $(n=22)$ pelas projeções oblíquas e somente $50 \%(n=11)$ pela ventrodorsal. As projeções ventrodorsal e oblíquas isoladamente não foram determinantes para diagnóstico em nenhum paciente deste estudo. Em 24,4\% (n=10) dos casos, foi observado mais que um local de compressão, tendo as projeções ventrodorsal e oblíquas auxiliado na definição do local de maior compressão em $50 \%$ e $70 \%$, respectivamente.

A análise de concordância Kappa entre A1 e A2 foi considerada forte para as projeções lateral $(0,78)$, ventrodorsal $(0,62)$ e oblíquas $(0,61)$. Entre A1 e A3, houve concordância razoável para a lateral $(0,36)$, forte para a ventrodorsal $(0,77) \mathrm{e}$ quase perfeita para as oblíquas $(0,85)$. Já entre A2 e A3, foi encontrada concordância moderada para 
as projeções lateral $(0,47)$ e oblíquas $(0,56)$ e forte para a projeção ventrodorsal $(0,71)$. O valor de concordância absoluta entre A1 e A2 nas projeções lateral, ventrodorsal e oblíquas foi de $97,56 \%, 90,24 \%$ e $82,92 \%$, respectivamente; para A1 e A3, esse resultado foi de $92,7 \%, 92,7 \%$ e 90,24\%; já para A2 e A3, foi de $95,1 \%, 90,24 \%$ e $78,04 \%$.

\section{DISCUSSÃO}

O espaço intervertebral mais comum de compressão medular por DDIV cervical em cães pode variar, conforme verificado em diferentes estudos. Cherrone et al. (2004) verificaram prevalência de compressões por DDIV tipo I em C2-C3. Outro estudo revelou maiores índices de compressão em C5-C6 (Hillman et al., 2009). Já para Ryan et al. (2008), o local mais afetado foi entre C6-C7. Neste estudo, no entanto, não houve um local com maior prevalência de compressão, demonstrando, assim, que a DDIV cervical não apresentou predisposição quanto ao espaço intervertebral na região cervical.

Não foi encontrada na literatura nenhuma recomendação acerca da sequência das projeções a serem feitas no exame de mielografia para a região cervical. Neste estudo, foi possível verificar que a projeção lateral foi a que mais identificou compressões, quando comparada às demais (oblíquas e ventrodorsal) $(\mathrm{P}<0,05)$. Dessa forma, recomenda-se que a projeção lateral deverá ser a primeira incidência a ser realizada após a injeção de contraste na região cervical.

Quanto às projeções ventrodorsal e oblíquas, mesmo não havendo diferença estatística na identificação de compressão, sugere-se que as oblíquas sejam realizadas após a projeção lateral. Para os autores deste trabalho, a projeção ventrodorsal deverá ser a última, pois todas as compressões detectadas por essa incidência foram observadas também nas oblíquas. Além disso, há necessidade de extubação do paciente, a fim de não haver sobreposição pelo traqueotubo, o que pode retardar a realização do exame (Aeschbacher, 2010). Gibbons et al. (2006) afirmaram que, devido à rápida absorção do contraste no espaço subaracnoide, o tempo entre cada projeção e a sequência delas pode afetar o diagnóstico e a qualidade das imagens.

Estudos em cães com DDIV toracolombar submetidos à mielografia demonstraram grande relevância das projeções oblíquas para localização da compressão, sendo especialmente importante para lesões lateralizadas (Tanaka et al., 2004; Gibbons et al., 2006; Zardo et al., 2010). Gibbons et al. (2006) verificaram, em cães com DDIV toracolombar, que as projeções oblíquas foram as mais determinantes para localização da lesão e sugeriram-nas como as primeiras projeções a serem realizadas após a injeção de contraste, seguidas pela ventrodorsal e pela lateral, ao contrário do que é proposto neste estudo, em que a projeção lateral deverá ser a primeira incidência, seguida das oblíquas e da ventrodorsal.

Gibbons et al. (2006) relataram lateralização em 99\% das compressões em cães com extrusão de disco ocasionada pela DDIV toracolombar. Esses resultados divergem dos encontrados neste trabalho, nos quais apenas 53,6\% (22/41) possuíam lesões lateralizadas. Uma hipótese levantada por esse grupo de estudo é que, devido ao fato de a relação diâmetro do canal vertebral/medula espinhal na região toracolombar ser menor do que na região cervical, o material extrudido tende a circunscrever a medula espinhal, conferindo padrões de lateralização no exame mielográfico. Como há mais espaço no canal vertebral e maior relação na região cervical (Brisson, 2010), o material tende a permanecer ventral à medula espinhal.

Devido ao alto índice em que a projeção lateral (40/41) foi capaz de localizar a compressão na medula espinhal cervical, não haveria necessidade de realizar as demais projeções, principalmente as oblíquas. Entretanto, mielografias nas projeções lateral e ventrodorsal podem não identificar compressões lateralizadas e intraforaminais, revelando a importância das projeções oblíquas também para a região cervical, conforme mencionaram Felts e Prata (1983). Todavia, neste estudo, pode-se verificar que as projeções oblíquas não foram determinantes para a identificação da compressão na medula espinhal, sendo sempre associadas às projeções lateral $(21 / 22)$ ou ventrodorsal $(11 / 22)$.

Além de identificar compressões lateralizadas e intraforaminais (Felts e Prata, 1983), as projeções oblíquas podem auxiliar na definição do espaço intervertebral a ser acessado cirurgicamente, quando houver mais de um local de compressão da medula espinhal, fato presenciado em sete dos 10 cães deste estudo. Outra contribuição das 
projeções oblíquas é auxiliar na escolha da técnica cirúrgica e na exploração cirúrgica do lado da compressão (Seim e Prata, 1982; Tanaka et al., 2004).

Embora o teste Kappa tenha revelado concordância razoável a moderada entre os avaliadores em alguns casos, a concordância absoluta foi considerada alta $(>90 \%)$ entre sete dos nove ensaios realizados. Segundo Stemler (2004), valores acima de $90 \%$ são considerados alta concordância. Essa baixa concordância entre os avaliadores para algumas projeções no teste Kappa pode ser explicada pela homogeneidade

e das amostras do estudo. Boudreau et al. (2018) relataram que o coeficiente de concordância do teste Kappa poderá ser reduzido quanto mais homogênea for a amostra. Assim, esses resultados podem ter sofrido interferência devido à sensibilidade e especificidade do teste.

Neste estudo, por ser retrospectivo, houve a necessidade de levantamento de dados de fichas clínicas, cujos resultados encontrados devem ser interpretados com cautela. Entretanto, todos os estudos epidemiológicos estão sujeitos a resultados subjetivos (Levine et al., 2007). O não sequenciamento (ordem) das imagens a serem radiografadas de cada paciente pode ter subestimado as projeções oblíquas e ventrodorsal. Por essa razão, sugerem-se estudos prospectivos para melhor avaliação da relevância dessas projeções com grupos e sequenciamentos mielográficos padronizados.

A relevância clínica deste estudo foi verificar que todas as projeções mielográficas são possíveis para identificação de compressão na medula espinhal em cães com DDIV cervical. E, com base na projeção mielográfica que mais revelou compressão, sugere-se que a projeção lateral deverá ser a primeira imagem radiografada, seguida pelas oblíquas direita ou esquerda e ventrodorsal.

\section{CONCLUSÃO}

Com base nos resultados deste estudo, pode-se concluir que todas as projeções mielográficas permitem identificar compressão da medula espinhal em cães com DDIV cervical, sendo a incidência lateral a que mais a revelou, seguida das oblíquas e ventrodorsal, estabelecendo-se, assim, uma proposta de sequenciamento das projeções mielográficas a serem realizadas para essa região.

\section{AGRADECIMENTOS}

Este estudo foi apoiado e financiado pelo Conselho Nacional de Desenvolvimento Científico e Tecnológico (CNPq), processo 307120/2017-1, e pela Coordenação de Aperfeiçoamento de Pessoal de Nível Superior, CAPES.

\section{REFERÊNCIAS}

AESCHBACHER, G. Anaesthesia. In: PLATT, S.R. Small animal neurology. Hannover: Schlütersche, 2010. p.73-85.

BOS, A.S.; BRISSON, B.A.; KYKAMP, S.G. et al. Accuracy, intermethod agreement, and interreviewer agreement for use of magnetic resonance imaging and myelography in small-breed dogs with naturally occurring first-time intervertebral disk extrusion. J. Am. Vet. Med. Assoc., v.240, p.969-977, 2012.

BOUDREAU, C.E.; DOMINGUEZ, A.E.; LEVINE, J.M. et al. Reliability of interpretation of neurologic examination findings for the localization of vestibular dysfunction in dogs. $J$. Am. Vet. Med. Assoc., v.252, p.830-838, 2018.

BRISSON, B.A. Intervertebral disc disease in dogs. Vet. Clin. N. Am. Small Anim. Pract., v.40, p.829-858, 2010.

CHERRONE, K.L.; DEWEY, C.W.; COATES, J.R.; BERGMAN, R.L. A retrospective comparison of cervical intervertebral disk disease in nonchondrodystrophic large dogs versus small dogs. J. Am. Anim. Hosp. Assoc., v.40, p.316-30, 2004.

DAVIDSON, H.C.; JOHNSTON, D.J.; CHRISTIAN, M.E.; HARNSBERGER, H.R. Comparison of radiographic image quality from four digitization devices as viwed on computer monitors. J. Digit. Imaging, v.14, p.24-29, 2001.

FELTS, J.F.; PRATA, R.G. Cervical disk disease in the dog: intraforaminal and lateral extrusions. J. Am. Anim. Hosp. Assoc., v.19, p.755-760, 1983. 
GIBBONS, S.E.; MACIAS, C.; DE STEFANI, A. et al. The value of oblique versus ventrodorsal myelographic views for lesion lateralization in canine thoracolumbar disc disease. J. Small Anim. Pract., v.47, p.658-662, 2006.

GRIFFIN, J.F.; LEVINE, J.M.; KERWIN, S.C. Canine thoracolumbar intervertebral disk disease: pathophysiology, neurologic examination and emergency medical therapy. Compend. Contin. Educ. Vet., v.31, p.1-13, 2009.

HANSEN, T.; SMOLDERS, L.A.; TRYFONIDOU, M.A. et al. The myth of fibroid degeneration in the canine intervertebral disc: a histopathological comparison of intervertebral disc degeneration in chondrodystrophic and nonchondrodystrophic dogs. Vet. Pathol., v.1, p.1-8, 2017.

HIGGINS T. An introduction to digital image processing. In: LANG, J.; MIDDLETON, A. Radiography of cultural material. Oxford: Butterworth-Heinemann, 1997. p.167-182.

HILLMAN, R.B.; KENGERI, S.S.; WATERS, D.J. Reevaluation of predictive factors for complete recovery in dogs with nonambulatory tetraparesis secondary to cervical disk herniation. J. Am. Anim. Hosp. Assoc., v.45, p.155-163, 2009.

LANDIS, J.R.; KOCH, G.G. The measurement of observer agreement for categorical data. Biometrics, v.33, p.159-174, 1977.

LEVINE, J.M.; LEVINE, G.J.; JOHNSON, S.I. et al. Evaluation of the success of medical management for presumptive thoracolumbar intervertebral disk herniation in dogs. Vet. Surg., v.36, p.482-491, 2007.

MCCARTNEY, W.T. Lumbar myelography in 79 dogs, using different puncture. Vet. Rec., v.141, p.417-419, 1997.
OLBY, N.J.; DYCE, J.; HOULTON, J.E.F. Correlation of plain radiographic and lumbar myelographic findings with surgical findings in thoracolumbar disc disease. J. Small Anim. Pract., v.35, p.345-350, 1994.

PRINCE, D.B.; ORTIZ, O. Myelography: from lipid-based to gadolinium-based contrast agents. Magn. Reson. Imaging Clin. N. Am., v.25, p.713724, 2017.

RYAN, T.M.; PLATT, S.R.; LLABRES-DIAZ, F.J. et al. Detection of spinal cord compression in dogs with cervical intervertebral disc disease by magnetic resonance imaging. Vet. Rec., v.163, p.11-15, 2008.

SHARP, N.J.H.; WHEELER, S.J. Diagnostic aids. In: _. Small animal spinal disorders diagnosis and surgery. 2.ed. Edinburgh: Elsevier Mosby, 2005. p.41-72.

STEMLER, S.E. A comparison of consensus, consistency, and measurement approaches to estimating interrater reliability. Pract. Assess. Res. Eval., v.9, p.1-11, 2004.

TANAKA, H.; NAKAYAMA, M.; TAKASE, K. Usefulness of myelography with multiple views in diagnosis of circumferential location of disc material in dogs with thoracolumbar intervertebral disc herniation. J. Vet. Med. Sci., v.66, p.827-833, 2004.

ZARDO, K.M.; PROVASI, A.; SELMI A.L.; ANDRADE NETO J.P. Contribuição das projeções oblíquas em mielografias de pequenos animais para a localização de lesões medulares causadas por processo degenerativo do disco intervertebral. Ciênc. Rural, v.40, p.2324-2331, 2010. 